\title{
Case Report \\ Brentuximab Vedotin Treatment for Primary Refractory Hodgkin Lymphoma
}

\author{
Hung-Bo Wu, ${ }^{1}$ Shyh-An Yeh, ${ }^{2}$ and Huei-Yung Chen ${ }^{3}$ \\ ${ }^{1}$ Division of Hematology and Oncology, Department of Medicine, Edah Hospital, No. 1, Yi-Da Road, Yen-Tsao District, \\ P.O. Box 824, Kaohsiung, Taiwan \\ ${ }^{2}$ Department of Radiation Oncology, Edah Hospital, Kaohsiung, Taiwan \\ ${ }^{3}$ Department of Nuclear Medicine, Edah Hospital, Kaohsiung, Taiwan
}

Correspondence should be addressed to Hung-Bo Wu; hbwuusa@yahoo.com

Received 15 July 2013; Accepted 12 September 2013

Academic Editors: R. Lowenthal and M. Nagasawa

Copyright (C) 2013 Hung-Bo Wu et al. This is an open access article distributed under the Creative Commons Attribution License, which permits unrestricted use, distribution, and reproduction in any medium, provided the original work is properly cited.

Up to $40 \%$ of patients with advanced Hodgkin lymphoma (HL) become refractory or relapsed after current standard chemotherapy, among which primary refractory HL confers a particularly poor outcome. With intensive salvage chemotherapy and autologous stem cell transplantation, the long-term remission rate for these patients was only $30 \%$, but more selective treatments with higher therapeutic index are needed. We report the experience of using a new anti-CD30 immunotoxin, brentuximab vedotin, in salvage treatment of a 30-year-old woman with primary refractory Hodgkin lymphoma. The patient presented with SVC syndrome due to the bulky mediastinal tumor and was confirmed to have classical Hodgkin lymphoma, nodular sclerosis type, stage IIIA. The tumor responded to induction chemotherapy transiently, but local progression was noted during subsequent cycles of treatment. Salvage radiotherapy to the mediastinal tumor, obtained no remission but was followed by rapid in-field progression and then lung metastasis. She declined stem cell transplantation and received salvage brentuximab vedotin (BV) therapy, which induced dramatic shrinkage of tumor without significant side effects. Serial followup of PET/CT imaging confirmed a rapid and continuous complete remission for 12 months. Although durability of the remission needs further observation, this case illustrates the excellent efficacy of brentuximab vedotin in primary refractory Hodgkin lymphoma.

\section{Background}

Despite recent improvement of therapy, the outcome of patients with refractory or relapsed Hodgkin lymphoma (RR-HL) remained compromised [1]. In order to improve the efficacy of induction treatment, the German Hodgkin Study Group (GHSG) developed a more intensive regimen (escalated BEACOPP), which achieved higher response rate (RR) and progression-free survival (PFS), but the overall survival (OS) was not improved due to increase of toxicities [2]. Salvage chemotherapy followed by autologous stem cell transplantation (ASCT) confers a long-term survival rate of $50 \%$ for patients with relapsed HL [3]. Unfortunately, this strategy had been less successful for the primary refractory HL, with poor long-term survival (only 30\%) but significant morbidities [4]. The optimal therapy for primary refractory $\mathrm{HL}$ remains undefined. Brentuximab vedotin (BV), an immunotoxin targeting cell-surface $\mathrm{CD} 30$ protein, had demonstrated efficacy in $\mathrm{HL}$ and was approved by FDA for treatment of HL relapse after ASCT or failure of two multiagent regimens and not candidates for ASCT. However, its role in the highly resistant primary refractory HL needs to be explored. Herein, we report a woman of primary refractory HL resistant to induction chemotherapy and salvage radiotherapy who achieved a rapid and persistent remission after BV treatment.

\section{Case Report}

A thirty-year-old female patient presented with dyspnea due to SVC syndrome. Chest CT scan showed bulky tumors over the mediastinum and left supraclavicular fossa, with right internal jugular vein thrombosis and right side pleural 


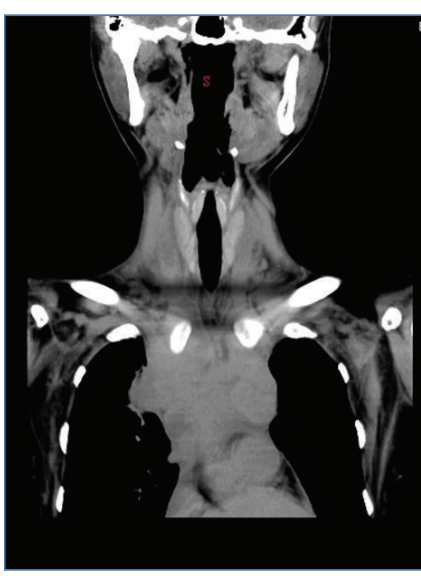

(a)

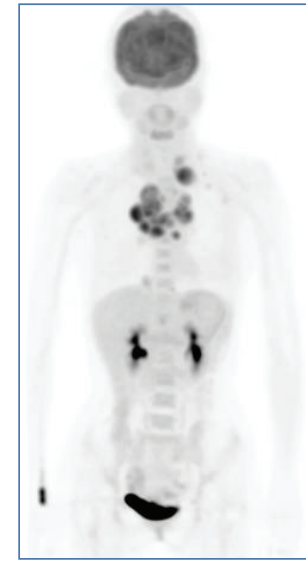

(b)

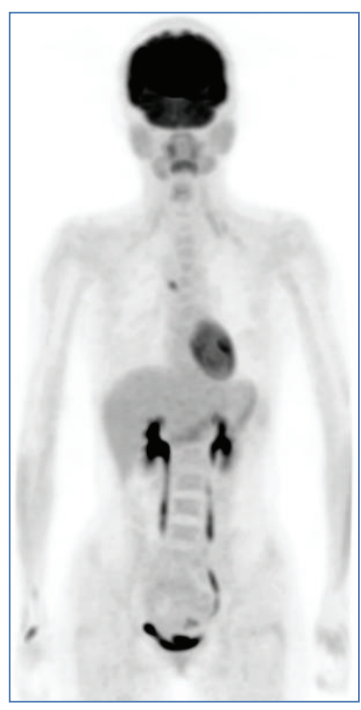

(c)

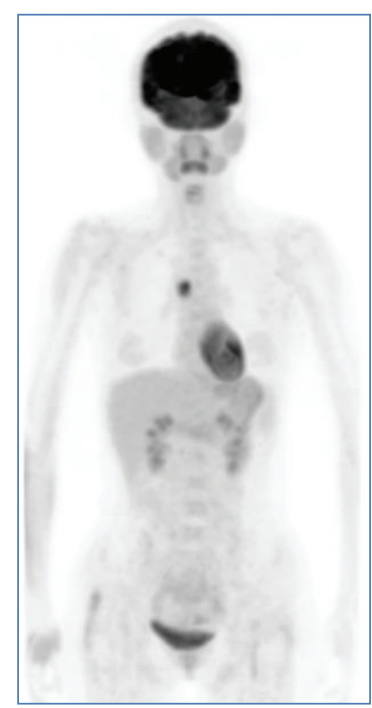

(d)

FIgURE 1: Images showing Hodgkin lymphoma refractory to induction chemotherapy. (a) Chest computerized tomography (CT) demonstrates the superior mediastinal tumor at diagnosis. (b) Positron emission tomography/computerized tomography (PET/CT) reveals involvement of right supraclavicular, left lower neck, mediastinal, epicardial, and retroperitoneal lymph nodes at initial diagnosis. (c) PET/CT after 4 cycles of induction chemotherapy shows partial remission with a residual uptake at right anterior mediastinum. (d) PET/CT after completion of 6 cycles of chemotherapy reveals relapse of the mediastinal tumor.

effusion (Figure 1(a)). Biopsy of the left neck tumor confirmed classical Hodgkin lymphoma, nodular sclerosing type. PET/CT study revealed involvement of right supraclavicular, left lower neck, mediastinal, epicardial, and retroperitoneal lymph nodes (Figure 1(b)).

Under diagnosis of classical Hodgkin lymphoma, stage IIIA, she received the standard induction chemotherapy with epirubicin, bleomycin, vinblastine, and dacarbazine (EBVD), with regression of neck tumors and SVC syndrome. After four cycles of chemotherapy, an interim PET/CT study showed partial remission with residual uptake over right anterior mediastinum (Figure 1(c)). The patient declined further histological study of the mediastinal mass and continued with the planned chemotherapy. After completion of six cycles of chemotherapy, local relapse of the mediastinal tumor was found in the posttreatment PET/CT study (Figure 1(d)).

Salvage radiotherapy was commenced for the progressive mediastinal tumor. The PET/CT scan images and CTsimulation images were fused for contouring target volumes. The involved lymphoid regions constituted the gross tumor volume. Isodose curves confirmed that at least $95 \%$ of the prescribed dose was delivered to the planning target volume. A total of $36 \mathrm{~Gy}$ in 20 daily fractions, 5 fractions per week, was delivered with intensity-modulated radiotherapy techniques. The patient tolerated it well, but CT imaging showed no remission of the mediastinal tumor followed by early relapse (Figures 2(a) and 2(b)).

Due to immediate relapse after induction chemotherapy and no response to salvage radiotherapy, primary refractory HL was impressed. Salvage chemotherapy and ASCT was suggested, but the patient hesitated due to concerns about anticipated toxicities with high-dose chemotherapy and ASCT. Two months later, left neck tumor enlarged significantly, the
PET/CT study revealed progressive mediastinal tumor with metastases to left lung, left axilla, and supraclavicular lymph nodes (Figure 3(a)). Biopsy of the left neck mass confirmed a recurrence of HL.

Recognizing the efficacy and side-effect profile of brentuximab vedotin, she opted for the new treatment. After approval by the National Department of Health, the drug was imported via the named-patient-program (Millennium/Takeda). The treatment was commenced at the recommended dose $\left(1.4 \mathrm{mg} / \mathrm{m}^{2}\right)$ every 3 weeks. The neck tumor regressed dramatically with minimal toxicity. Follow-up PET/CT scans after 4 and 9 cycles of BV showed CR of the tumor (Figures $3(\mathrm{~b})$ and $3(\mathrm{c})$ ). The most recent PET/CT scanning confirmed continuous remission of the tumor for 11 months. The patient is in good health and receives close monitoring of remission status, with ASCT as an option for further salvage.

\section{Discussion}

Optimal management of RR-HL is a difficult clinical challenge with three conventional options of salvage treatment: radiotherapy, chemotherapy, and stem cell transplantation.

For selected patients with confirmed local relapse, salvage radiotherapy is a very effective treatment modality with nearly $80 \%$ CR rate and $65 \%$ long-term local control in the GHSG report $[5,6]$. But most patients will undergo secondline chemotherapy in order to obtain a second remission. Phase II trials of various salvage chemotherapy regimens had reported RR around $75 \%$, with CR rate around $40 \%$. However, there are no direct comparison of different regimens and no consensus on the standard second-line chemotherapy [7]. 


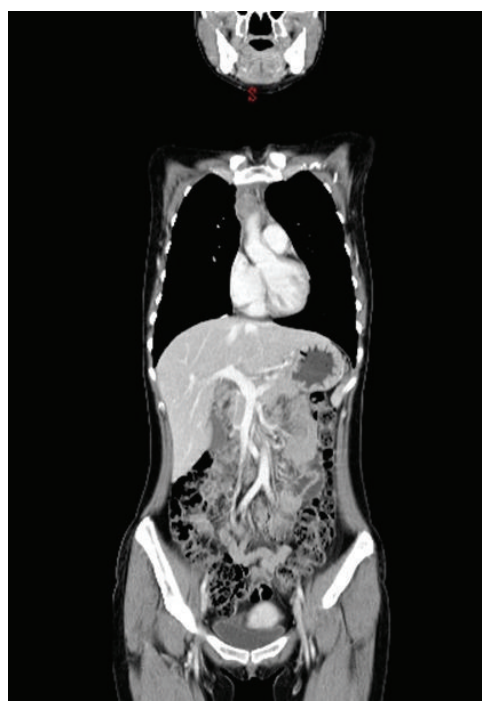

(a)

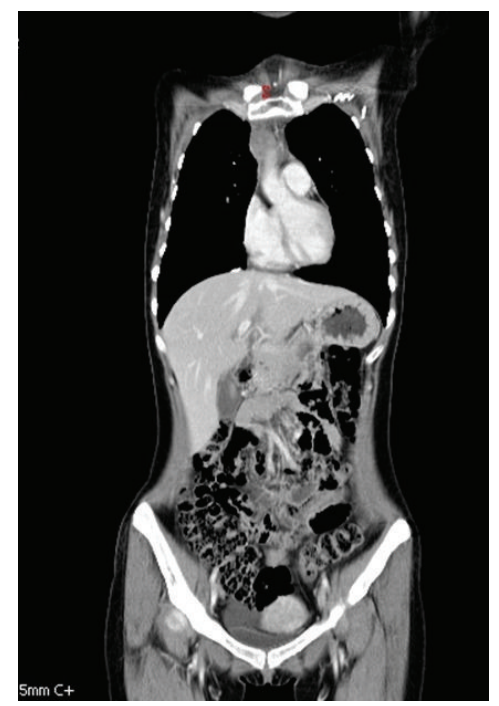

(b)

FIGURE 2: Mediastinal tumor refractory to salvage radiotherapy. (a) CT shows relapsed superior mediastinal tumor before radiotherapy. (b) CT reveals progression of the superior mediastinal tumor 2 months after completion of radiotherapy.

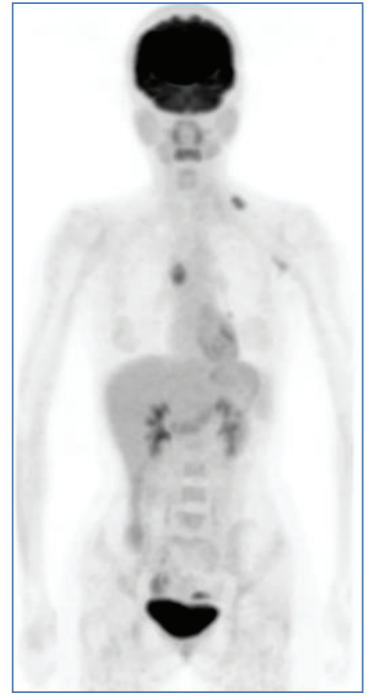

(a)

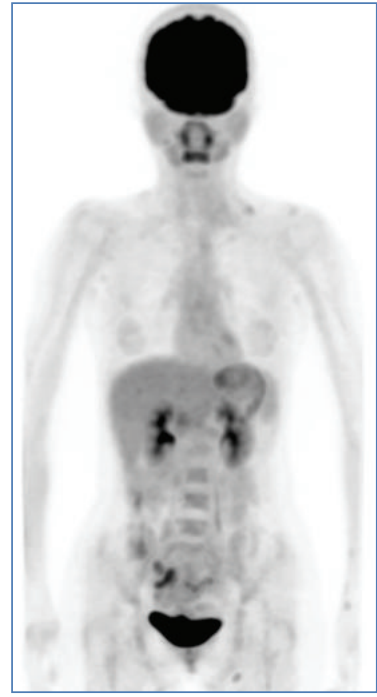

(b)

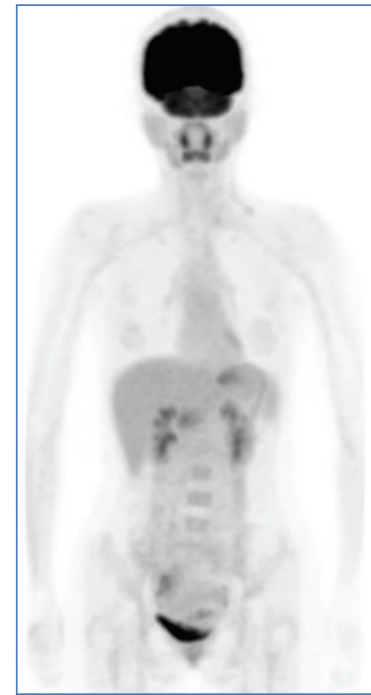

(c)

Figure 3: Serial PET/CT images before and after brentuximab vedotin treatment. (a) Before treatment, showing mediastinal tumor progression, with metastases to left lung, left axillary, and supraclavicular lymph nodes. (b) Near-complete remission after 4 cycles of treatment, with a faint uptake over left lower neck. (c) Complete remission after 9 cycles of treatment.

For patients with sensitive relapse, high-dose chemotherapy followed by ASCT is the current standard treatment based on two randomized phase III studies by the British National Lymphoma Investigation and the GHSG, reporting 3-year PFS around 50\%. However, chemorefractory patients were underrepresented in these trials due to exclusion of most patients with rapid progression $[8,9]$.

Among the adverse prognostic factors identified in patients with RR-HL, remission duration after initial treatment was particularly important. In a report of salvage chemotherapy for RR-HL by the NCI Italy, primary refractory
HL has the poorest eight-year survival of $8 \%$ (versus $28 \%$ for early relapse and $54 \%$ for late relapse) [10]. The fiveyear-survival rates of patients in the GHSG cohort were $26 \%$ for the primary refractory (progression during induction treatment or within 3 months after the end of treatment), $46 \%$ for the early relapse (between 3 and 12 months), and $71 \%$ for the late relapse (after 12 months) [11]. Patients achieved tumor remission after salvage chemotherapy then underwent ASCT which yielded a higher OS of $42 \%$ in the GHSG report. However, further exploratory landmark analysis suggested the favorable result of ASCT might be due 
to exclusion of poor-risked patients with refractory disease, poor-performance status, older age, insufficient stem cell harvest, or life-threatening toxicity on salvage treatment [11]. The GELA H89 study was designed to explore prospectively the strategy of early intensified chemotherapy followed by HDC/ASCT in patients of RR-HL and found the 5-year OS in primary refractory HL to be significantly lower than in other RR-HL patients (30\% versus $74 \%, P=0.0001$ ) [4].

Considering the observed tradeoff between efficacy and toxicities of intensified chemotherapy and the suboptimal outcome of ASCT in refractory cases, more selective drug with higher therapeutic index is clearly needed for the poorrisked HL. Brentuximab vedotin (SGN 35) is a chimeric antiCD30 antibody conjugated by a protease-cleavable linker to a microtubule-disrupting agent (monomethyl auristatin E; MMAE). After binding to cell-surface CD30, the MMAE is internalized, traffics to the lysosome, and then is released to disrupt microtubules, and to induce cell-cycle arrest and apoptosis. In the phase I dose escalation trial of 45 patients with relapsed/refractory CD30+ hematological malignancies, tumor regression was observed in $86 \%$ of cases [12]. The subsequent pivotal phase II trial (SG035-0003) in 102 patients of Hodgkin lymphoma with post-ASCT relapses achieved a $75 \%$ response rate $(34 \% \mathrm{CR})$ and $96 \%$ disease control rate [13]. Considering the high proportions of poor risk features in these patients-primary refractory (in 72\%), refractory to salvage therapy (in 42\%), and early relapse after ASCT (in $71 \%$ ) - the new drug seemed to be effective across all the high-risk groups. These favorable results led to accelerated approval by the US FDA in August 2011.

The safety profiles for BV are favorable compared with conventional cytotoxic chemotherapy. More common side effects are usually mild and include cough, fatigue, pyrexia, nausea, and peripheral neuropathy. The most clinical relevant toxicity is peripheral neuropathy, which is usually of sensory type, with an $80 \%$ spontaneous improvement rate. A rare but severe side effect of progressive multifocal leukoencephalopathy was reported recently in 3 cases (among 2000+ patients). Clinician should stay alert to and stop the medication with any newly onset CNS signs and symptoms.

Exciting reports about brentuximab vedotin are continuously emerging. In patients with post-ASCT relapse, a recent study confirmed the superior survival with BV than any other salvage treatments (median: 91.49 mo versus 27.99 mo, $P=0.0004)$. In this report, patients achieving CR after $\mathrm{BV}$ had a prolonged progression-free survival of 29 months [14]. Other exciting reports included higher CR rate of BV (34\%) than ASCT (15\%) in RR-HL [15], a role in facilitation of reduced-intensity allotransplantation [16] and effective salvage of relapse after allo-SCT [17].

Many researches are currently ongoing to explore the expanding roles of this new drug in management of HL and other $\mathrm{CD} 30+$ malignancies. However, the role of $\mathrm{BV}$ in salvage treatment of primary refractory HL is an underexplored area which needs further clinical studies. This case scenario shows good efficacy of BV in primary refractory $\mathrm{HL}$ and suggests the potential use in this poor-risk group. Hopefully, with optimal incorporation of BV into current treatment modalities, the survival of primary refractory and other poor-risk Hodgkin lymphoma will be further improved.
Abbreviations
BV: $\quad$ Brentuximab vedotin
RR-HL: Relapsed or refractory Hodgkin lymphoma
GHSG: German Hodgkin Study Group
PFS: Progression-free survival
OS: Overall survival
ASCT: Autologous stem cell transplantation
CR: Complete remission.

\section{Consent}

Written informed consent was obtained from the patient for publication of this case report and accompanying images. A copy of the written consent is available for review.

\section{Conflict of Interests}

The authors declare that they have no conflict of interests.

\section{Authors' Contribution}

Hung-Bo Wu was responsible for clinical management of the patient and drafting of the paper. Shyh-An Yeh participated in patient management and drafting of the paper. Huei-Yung Chen carried out the PET/CT imaging, interpretation, and helped in preparation of the paper. All authors read and approved the final paper.

\section{References}

[1] J. Kuruvilla, "Standard therapy of advanced Hodgkin lymphoma," Hematology, pp. 497-506, 2009.

[2] J. M. Connors, "More is not necessarily better when treating Hodgkin's lymphoma," Journal of Clinical Oncology, vol. 29, no. 32, pp. 4215-4216, 2011.

[3] A. Sureda, M. Constans, A. Iriondo et al., "Prognostic factors affecting long-term outcome after stem cell transplantation in Hodgkin's lymphoma autografted after a first relapse," Annals of Oncology, vol. 16, no. 4, pp. 625-633, 2005.

[4] C. Fermé, N. Mounier, M. Diviné et al., "Intensive salvage therapy with high-dose chemotherapy for patients with advanced Hodgkin's disease in relapse or failure after initial chemotherapy: results of the Groupe d'Études des Lymphomes de l'Adulte H89 trial," Journal of Clinical Oncology, vol. 20, no. 2, pp. 467475, 2002.

[5] A. Josting, L. Nogová, J. Franklin et al., "Salvage radiotherapy in patients with relapsed and refractory Hodgkin's lymphoma: a retrospective analysis from the German Hodgkin Lymphoma Study Group," Journal of Clinical Oncology, vol. 23, no. 7, pp. 1522-1529, 2005.

[6] B. Campbell, A. Wirth, A. Milner, J. Di Iulio, M. MacManus, and G. Ryan, "Long-term follow-up of salvage radiotherapy in Hodgkin's lymphoma after chemotherapy failure," International Journal of Radiation Oncology Biology Physics, vol. 63, no. 5, pp. 1538-1545, 2005. 
[7] J. Kuruvilla, A. Keating, and M. Crump, "How I treat relapsed and refractory Hodgkin lymphoma," Blood, vol. 117, no. 16, pp. 4208-4217, 2011.

[8] D. C. Linch, D. Winfield, A. H. Goldstone et al., "Dose intensification with autologous bone-marrow transplantation in relapsed and resistant Hodgkin's disease: results of a BNLI randomised trial," The Lancet, vol. 341, no. 8852, pp. 1051-1054, 1993.

[9] N. Schmitz, B. Pfistner, M. Sextro et al., "Aggressive conventional chemotherapy compared with high-dose chemotherapy with autologous haemopoietic stem-cell transplantation for relapsed chemosensitive Hodgkin's disease: a randomised trial," The Lancet, vol. 359, no. 9323, pp. 2065-2071, 2002.

[10] V. Bonfante, A. Santoro, S. Viviani et al., "Outcome of patients with Hodgkin's disease failing after primary MOPP- ABVD," Journal of Clinical Oncology, vol. 15, no. 2, pp. 528-534, 1997.

[11] A. Josting, U. Rueffer, J. Franklin, M. Sieber, V. Diehl, and A. Engert, "Prognostic factors and treatment outcome in primary progressive Hodgkin lymphoma: a report from the German Hodgkin Lymphoma Study Group," Blood, vol. 96, no. 4, pp. 1280-1286, 2000.

[12] A. Younes, N. L. Bartlett, J. P. Leonard et al., "Brentuximab vedotin (SGN-35) for relapsed CD30-positive lymphomas," New England Journal of Medicine, vol. 363, no. 19, pp. 1812-1821, 2010.

[13] A. Younes, A. K. Gopal, S. E. Smith et al., "Results of a pivotal phase II study of brentuximab vedotin for patients with relapsed or refractory Hodgkin's lymphoma," Journal of Clinical Oncology, vol. 30, no. 18, pp. 2183-2218, 2012.

[14] M. S. Karuturi, S. Arai, R. W. Chen et al., "Overall survival benefit for patients with relapsed Hodgkin lymphoma treated with brentuximab vedotin after autologous stem cell transplant," in Proceedings of the ASH Annual Meeting Abstracts, vol. 3701, 2012.

[15] A. Gualberto, A. Chi, and Y. Liu, "Activity of the investigational antibody-drug conjugate brentuximab vedotin (SGN-35) in patients with relapsed or refractory Hodgkin lymphoma or systemic anaplastic large-cell lymphoma: comparisons with meta-analyses of historical control chemotherapy data," in Proceedings of the ASH Annual Meeting Abstracts, vol. 4975, 2012.

[16] R. W. Chen, J. M. Palmer, S. H. Thomas et al., "Brentuximab vedotin, (SGN-35) enables successful reduced intensity allogeneic hematopoietic cell transplantation in relapsed/refractory Hodgkin lymphoma," Blood, vol. 119, no. 26, pp. 6379-6381, 2012.

[17] A. K. Gopal, R. Ramchandren, O. A. O'Connor et al., "Safety and efficacy of brentuximab vedotin for Hodgkin lymphoma recurring after allogeneic stem cell transplantation," Blood, vol. 120, no. 3, pp. 560-568, 2012. 


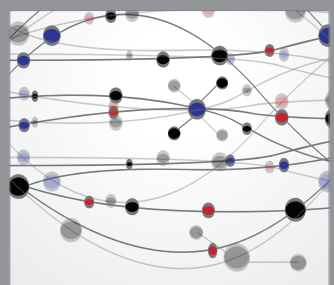

The Scientific World Journal
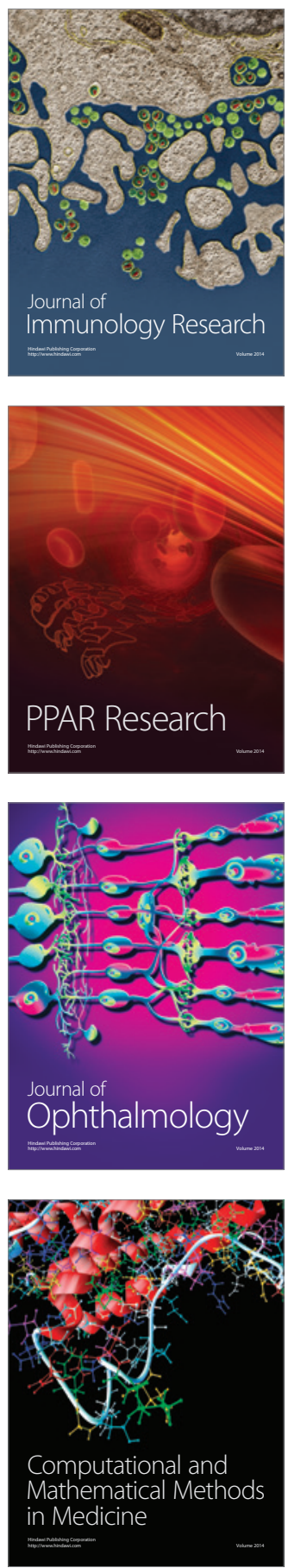

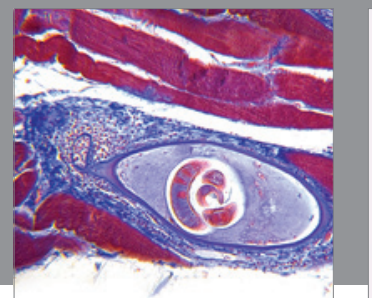

Gastroenterology

Research and Practice
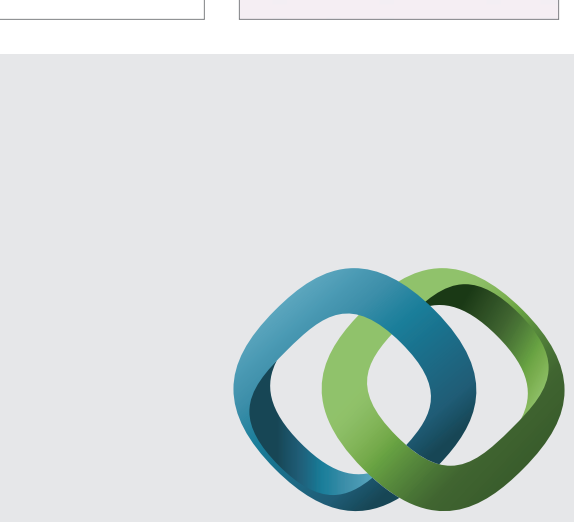

\section{Hindawi}

Submit your manuscripts at

http://www.hindawi.com
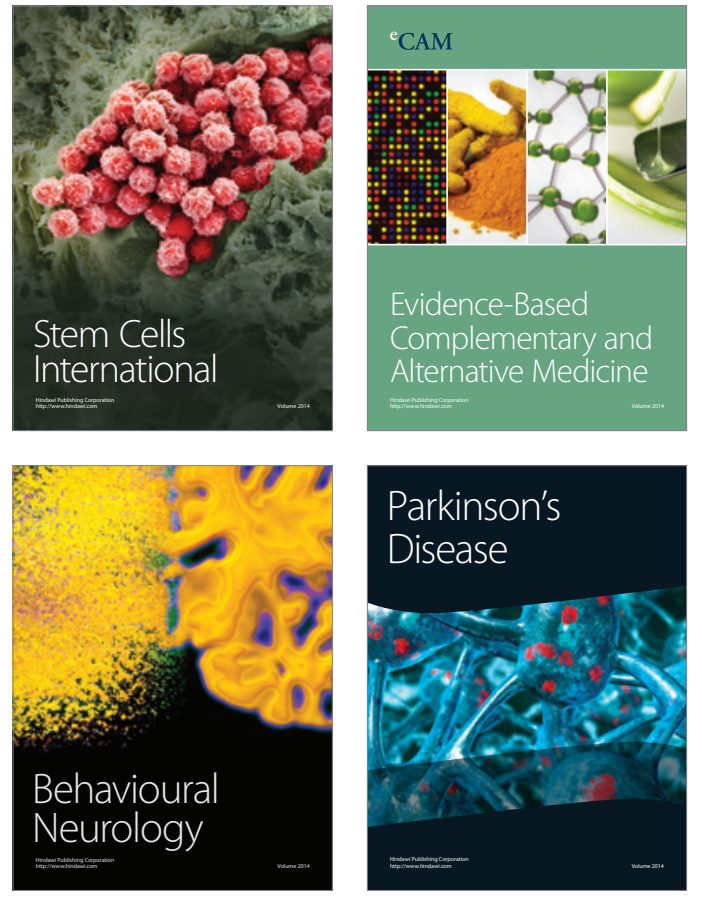
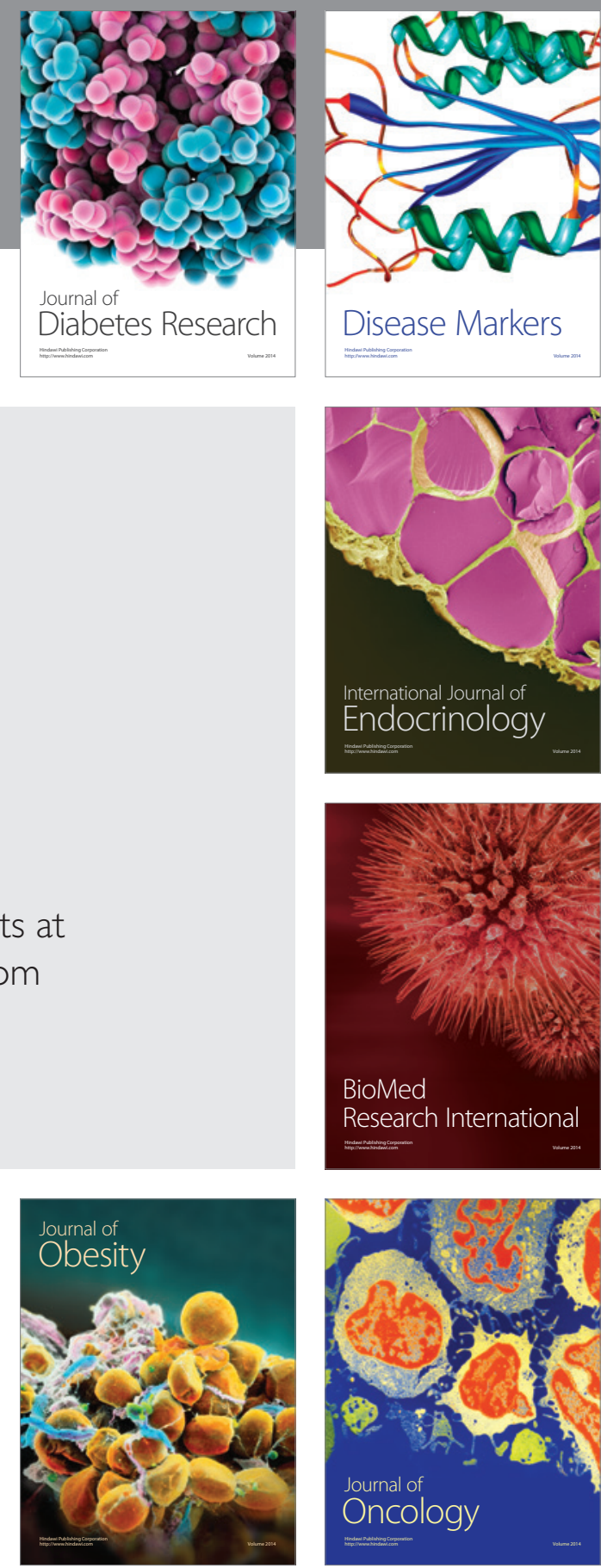

Disease Markers
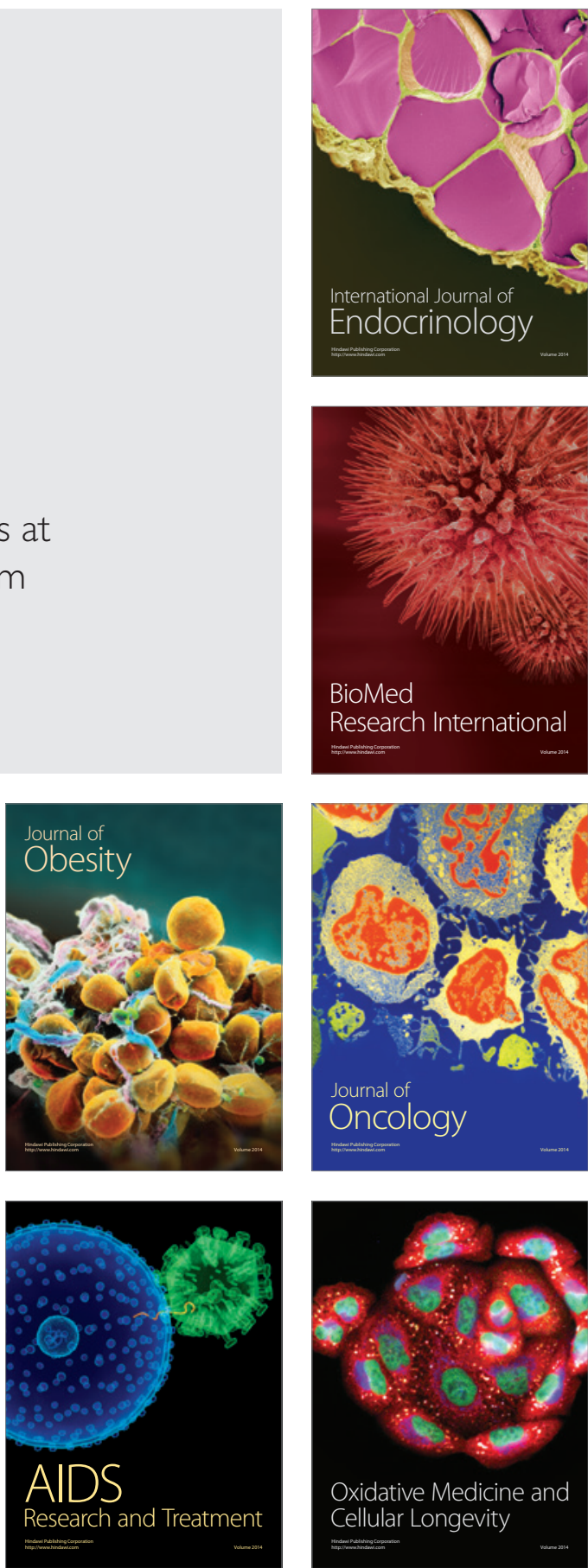